\title{
Prevalence and prognosis of acutely ill patients with organ failure at arrival to hospital: protocol for a systematic review
}

Peter Bank Pedersen ${ }^{1 *}$, Asbjorn Hrobjartsson ${ }^{2}$, Daniel Lykke Nielsen ${ }^{3}$, Daniel Pilsgaard Henriksen ${ }^{4}$, Mikkel Brabrand ${ }^{5,6}$ and Annmarie Touborg Lassen ${ }^{1}$

\begin{abstract}
Background: Acutely ill patients are a heterogeneous group, and some of these suffer from organ failure. As the prognosis of organ failure improves with early treatment, it is important to identify these patients as early as possible. Most studies on organ failure have been performed in intensive care settings, or on selected groups of patients, where a high prevalence and mortality have been reported. Before patients arrive to the intensive care unit, or the general ward, most of them have passed through the emergency department (ED), where diagnosis and treatment has been initiated. The prevalence and prognosis of acutely ill patients, with organ failure, at arrival have been studied in some selected groups, but methods and results differ. This systematic review aims to identify, summarize, and analyze studies of prevalence and prognosis of new onset organ failure in acutely ill undifferentiated patients, at arrival to hospital. The result of the review will assist physicians working in an ED, when assessing patients' risk of organ failure and their associated prognosis.

Methods: The information sources used are electronic databases, PubMed, Cochrane Library, EMBASE, and CINAHL; references in included studies and review articles; and authors' personal files. One author will perform the title and abstract screening and exclude obviously ineligible studies. By an independent full-text screening, two authors will decide on the eligibility for the remaining studies. Eligible studies will include an unselected group of acutely ill adult patients at arrival to hospital, with one or more organ failures (respiratory, renal, cerebral, circulatory, hepatic, or coagulation failure). Included studies will have assessed the prevalence or prognosis, defined as mortality or ICU transfer, of new onset organ failure. From included studies, bibliographical and study description data, patient characteristics, and data related to prevalence of organ failure and prognosis will be extracted. We will assess risk of bias in included studies using the Quality in Prognosis Studies tool for prognostic studies and the Newcastle-Ottawa Scale for observational studies. We expect heterogeneity and to conduct a qualitative synthesis of the results. If, however, heterogeneity is low, we will conduct a random effects meta-analysis stratified by basic study design.
\end{abstract}

Discussion: This review will summarize and analyze studies of prevalence and prognosis of acutely ill patients, with organ failure at arrival to hospital, assist ED physicians assessing the risk of organ failure in unselected patients, and guide recommendations for further research.

\section{Systematic review registration: PROSPERO CRD42017060871}

Keywords: Organ failure, Emergency department, Emergency medicine, Acute medicine, Arrival, Prevalence, Prognosis

\footnotetext{
* Correspondence: Peter.Bank.Pedersen@rsyd.dk

'Department of Emergency Medicine, Institute of Clinical Research, University

of Southern Denmark and Odense University Hospital, DK-5000 Odense C,

Denmark

Full list of author information is available at the end of the article
} 


\section{Background}

Patients arriving with an acute condition are a heterogeneous group, and the initial step is to identify the critically ill. Some patients have organ failures based on different etiologies, affecting different organs with diverse severity, and identifying these is also a priority. Most of the existing knowledge regarding organ failure has been obtained in intensive care settings or on selected groups of patients, not on undifferentiated patients at the hospital doorstep, but later on.

Studies on patients in intensive care units (ICU) have reported a high prevalence of at least one organ failure $(51-72 \%)$ at some point during the ICU stay. Respiratory organ failure is most prevalent, affecting as many as $87 \%$ organ failure patients. Prognosis described as in-hospital, short-term, and long-term mortality depends on the severity of organ dysfunction, number of failing organs, and the specific organ affected, and 5-year mortality is described at approximately $60 \%$ [1-4].

Outside the ICU, prevalence of organ failure, in unselected ward patients or after injury in US trauma and nontrauma centers, is described at approximately $7-14 \%$. In undifferentiated patients with shock, the presence of organ failures has been described as $70 \%$, at arrival to the emergency department. Organ failure is associated with increased in-hospital mortality, which increases with each additional organ failure, ranging from 12 to $60 \%$ with one to more than three organ failures [5-7].

Organ failure or the number of organ failures is also a risk factor in patients with severe sepsis [8]. There has been demonstrated an association between increasing number of organ failures and increased in-hospital, short-term, and long-term mortality and an association with ICU transfer from the emergency department. Likewise, it has been demonstrated that presence of clinically recognizable signs of organ failure results in better treatment compared to organ-specific laboratory values. The most common organ failures have been described as cardiovascular, renal, and respiratory [9-13]. Sepsis survivors have showed rates of persistent and long-term ( $\geq 90$ days) organ dysfunction to be no less than 27 and $21 \%$ respectively [14].

Patients suffering from organ failure have to be identified and treated as early as possible. Before patients arrive to the ICU or general ward, most have passed the emergency department (ED) where the ED doctors have treated or started treatment due to acute illness. Furthermore, identifying patients at greatest risk for chronic organ failure is important, because it will allow early identification of susceptible patients which needs preventive interventions [14]. By this systematic review, we wish to identify, summarize, and analyze studies on the prevalence and prognosis of new onset organ failure in acutely ill undifferentiated patients at arrival on the hospital doorstep.

\section{Methods/design}

We plan to conduct a systematic review. This protocol is reported according to the Preferred Reporting Items for Systematic Reviews and Meta-Analysis Protocols (PRISMA-P) 2015 statement (see checklist in Additional file 1) and developed with inspiration from the Cochrane Handbook for Systematic Reviews of Interventions [15-17].

\section{Objectives}

Our overall aim is to assess the prevalence and prognosis of new organ failures in acutely ill patients at arrival to hospital. Our main research objective is to assess (1) the prevalence of new onset organ failures in acutely ill patients at arrival to hospital and (2) the prognosis of patients with newly onset organ failure at arrival to hospital.

\section{Eligibility criteria \\ Study designs}

We will include observational studies (cross-sectional studies, prospective and retrospective cohort studies, and case-control studies), and randomized and nonrandomized controlled trials, assessing the prevalence of new organ failure, or prognosis of acutely ill patients at hospital arrival. We will exclude case reports and studies with less than 100 patients.

\section{Participants/population}

Eligible studies will include an unselected group of acutely ill adult patients. Studies on selected groups of patients, such as specific conditions or diseases, will be excluded. Studies not restricted to adults are eligible and will be included in our analyses, provided that separate adult-data evaluation is achievable.

Studies on acutely ill patients, with one or more of the following organ failures, will be included: respiratory failure, renal failure, cerebral failure, circulatory failure, hepatic failure, or coagulation failure.

\section{Outcomes}

Studies that assess the prevalence of one or more organ failures, disregarding how prevalence has been defined or measured, will be included. Studies that have assessed prognosis, defined as mortality (short-term, long-term, and all-cause mortality and organ failure-specific mortality) or ICU transfer, will be included as well. Included studies may have dissimilar definitions of organ failures and may define short-term and longterm mortality by different thresholds. We will adhere to the organ failure definition as reported in the individual study and aim to extract mortality data as close as possible for 30-day and 1-year mortality. 


\section{Setting}

We will include studies of patients which arrive to hospital at an emergency department, a trauma centre, a general ward, an acute medical unit, or other entrances for acutely ill patients. Studies where patients arrive directly at an intensive care unit are excluded.

\section{Language}

Studies published or conducted in English, or other languages the author group are able to read, will be included. Study titles in other languages will be listed and provided as an appendix if they seem relevant.

\section{Information sources}

Information sources used are electronic databases not restricted to a specific period of time, references in included studies and review articles, and authors' personal files. The databases we will use are PubMed, Cochrane Library, EMBASE, and CINAHL, and the protocol database, PROSPERO, will be searched for ongoing or recently completed systematic reviews on similar topics.

\section{Search strategy}

Search strategy for the databases will be developed iteratively by the input from all the members of the project team and with the help from an information specialist from The Medical Research Library at the University of Southern Denmark in a face-to-face meeting.

We will systematically scan the reference lists of included studies or reviews for eligible studies and inspect authors' personal files. We will not contact content experts for a list of possibly eligible studies.

When a search has been performed at one electronic database, the same search will be performed at the other databases and only adapt the exact subjects and syntax, which fit in that particular database. Just before finalizing the review, the search will be updated to ensure the most recent relevant studies are included. To increase transparency, a research record table (Table 1) will be included.

Draft PubMed:

Organ failure

Organ failure OR organ failures

Organ failure OR organ failures OR organ dysfunction Organ failure OR organ failures OR organ dysfunction OR organ dysfunctions

Organ failure OR organ failures OR organ dysfunction OR organ dysfunctions OR organ system dysfunction

Table 1 Research record table

Information source Date searched Search References Comments
Organ failure OR organ failures OR organ dysfunction OR organ dysfunctions OR organ system dysfunction AND emergency department Organ failure OR organ failures OR organ dysfunction OR organ dysfunctions OR organ system dysfunction AND (emergency department OR emergency room) Organ failure OR organ failures OR organ dysfunction OR organ dysfunctions OR organ system dysfunction AND (emergency department OR emergency room OR acute medical unit)

Organ failure OR organ failures OR organ dysfunction OR organ dysfunctions OR organ system dysfunction AND (emergency department OR emergency room OR acute medical unit OR non-ICU)

\section{Study records \\ Data management}

References, from the literature search, will be exported to the software program "Endnote," where a check for duplicates is performed. Afterwards, references are transferred to the software program "Covidence.org" for further processing. An internal study audit will be performed on studies conducted by identical authors to avoid double counting, and studies on identical data will be compared to clarify inconsistencies.

\section{Selection process}

PBP will perform title and abstract screening and exclude obviously ineligible studies. Other studies will be read in full length, independent, and in duplicate by two review authors, PBP and DLN. Subsequently, in agreement, the two review authors will decide whether the study meets inclusion criteria. The proportion of agreement is presented in the final review. Disagreements will be discussed at a face-to-face meeting, and in the case of continued disagreements, AH and ATL's point of view will decide for the inclusion. Reasons for excluding full-text studies will be documented.

\section{Data collection process and data items}

From included studies, we will extract bibliographical and study description data, patient characteristics, and data related to prevalence of organ failure and prognosis. Data extraction will be performed, independent and in duplicate by two reviewers, using predefined data fields. After pilot data extraction involving two to three studies, the data extraction sheet will be revised. In case of discrepancies, the rest of the review group is involved. In the absence of complete description of data or outcomes, we will attempt to establish contact to the authors by e-mail, as many as three times. 


\section{Outcomes and prioritization}

The primary outcome will be number of organ failures, and patients with organ failures at arrival (first recorded vital and laboratory values within $24 \mathrm{~h}$ or as close as possible) per 1000 visits. Organ failures studied are respiratory, circulatory, renal, hepatic, coagulatory, and cerebral, with any definition per original paper.

The secondary outcome, for patients with organ failure, is prognosis, assed by proportion and relative risk of transfer to ICU and/or 30-day (or as close as possible) and 1-year (or as close as possible) all-cause mortality.

\section{Risk of bias in individual studies}

To assess the risk of bias within included studies, the Quality in Prognosis Studies (QUIPS) tool for prognostic studies, the Newcastle-Ottawa Scale (NOS) for observational studies, and the Cochrane Risk of Bias Tool (CRBT) for randomized controlled trials will be used. The QUIPS tool rates six bias domains: study participation, study attrition, prognostic factor measurement, outcome measurement, study confounding, and statistical analysis and reporting, as having high, moderate, or low risk of bias [18]. The NOS evaluate selection, comparability, and outcome in case-control and cohort studies by assigning stars [19]. The CRBT rates the following: selection bias, performance bias, detection bias, attrition bias, reporting bias, and other biases as low, high, or unclear [20]. Two independent reviewers, PBP and DLN, will assess every included study for bias; neither of the assessors is blinded to the studies, and disagreements will be resolved by discussion and eventually by consulting ATL and AH from the review group.

\section{Data synthesis}

On basis of scoping searches conducted in preparation for this review, we do not anticipate to conduct a metaanalysis, as considerable diversity in studies to be included is expected. In any case, we will explore reasons for the expected heterogeneity according to clinical (participants and outcomes) and methodological (design and risk of bias) characteristics. Statistical test for heterogeneity will be performed (Cochran's Q), and degree of heterogeneity will be described with the $I^{2}$ statistic. In the case of substantial and statistically significant heterogeneity ( $I$-square $>60 \%, P<0.10)$, a meta-analysis with the aim of assessing a weighted average will not be performed [21].

If heterogeneity is low, contrary to expectation, we will conduct a random effects meta-analysis, stratified by basic study design. We will present both synthetic and analytic views and try to explain heterogeneity by study characteristics and population characteristics.

We anticipate to perform a qualitative synthesis of the included studies (reporting median and interquartile results) based on tables and graphs to sum up results and findings. The synthesis will include basic study characteristics, results (including heterogeneity), risk of bias assessments, and explorations of reasons for heterogeneity [22].

In subgroups of patients with organ failure following trauma, bleeding, cardiac failure, or sepsis, sub-group analysis will be performed based on patient characteristics, types, and numbers of organ failure.

\section{Confidence in cumulative evidence}

We will assess certainty of evidence provided by our review, inspired by the Grading of Recommendations Assessment (GRADE approach) depending on the basic design of the included studies and any downor upgrading decisions as high, moderate, low, or very low. We will use the following assessment criteria when considering downgrading the certainty of the evidence: risk of bias, inconsistency, indirectness, imprecision, and publication bias to decide how to grade the certainty of evidence [23, 24]. As our study is observational by nature and do not address effect, we will not upgrade evidence based on standard criteria. The assessments will be performed by two review authors, PBP and DLN, independently, and disagreements will be resolved by discussion.

\section{Amendments}

In case of protocol amendments, a table will be added with a description of every change, the rationale, and a date. Changes will not be incorporated into the protocol. Significant amendments will be registered in the PROSPERO register, and approval by all authors is required prior to registration. The review will contain a section describing differences between protocol and review.

\section{Discussion}

The overarching goal of this review is to summarize data on prevalence and prognosis for acutely ill patients with organ failure at arrival to hospital. As early identification and treatment of these patients improves prognosis, systematic knowledge of the epidemiology might help clinical treatment in the emergency department. This has the potential to change the direct clinical management, as well as provide information of where to increase resources for the immediate management of acutely ill patients with organ failure.

This systematic review is planned to be published in a peer-reviewed journal.

\section{Additional file}

Additional file 1: PRISMA-P 2015 checklist. (DOCX 35 kb) 


\section{Abbreviations}

CRBT: Cochrane Risk of Bias Tool; ED: Emergency department; GRADE: Grading of Recommendations Assessment; ICU: Intensive care unit; NOS: Newcastle-Ottawa Scale; PRISMA-P: Preferred Reporting Items for Systematic Reviews and Meta-Analysis Protocols; QUIPS: Quality in Prognosis Studies

\section{Acknowledgements}

An information specialist from The Medical Research Library, Videncentret, part of The University Library of Southern Denmark, will help in conducting the search strategy and obtaining the articles.

\section{Funding}

No external funding has been received for this review. PBP was supported by the University of Southern Denmark and the Region of Southern Denmark, but they are not involved in any aspect of this systematic review and have no influence on the study results or publication and no role in the study design, data collection, or analysis. ATL received an unrestricted grant from the private philanthropic fund TrygFonden given to the University of Southern Denmark.

\section{Availability of data and materials}

Not applicable.

\section{Authors' contributions}

PBP, AH, DLN, DPH, MB, and ATL took part in the draft and formulated the manuscript of the protocol. PBP, AH, and ATL contributed in the development of the inclusion criteria and the analysis strategy. PBP composed the search strategy, with inputs from AH and ATL. PBP and DLN took part in the selection of studies included in the final review. PBP is the guarantor. All authors provided feedback and read and approved the final manuscript.

\section{Ethics approval and consent to participate}

Not applicable.

\section{Consent for publication}

Not applicable.

\section{Competing interests}

The authors declare that they have no competing interests.

\section{Publisher's Note}

Springer Nature remains neutral with regard to jurisdictional claims in published maps and institutional affiliations.

\section{Author details}

${ }^{1}$ Department of Emergency Medicine, Institute of Clinical Research, University of Southern Denmark and Odense University Hospital, DK-5000 Odense C, Denmark. ${ }^{2}$ Centre for Evidence-Based Medicine, University of Southern Denmark and Odense University Hospital, DK-5000 Odense C, Denmark. ${ }^{3}$ Department of Emergency Medicine, Odense University Hospital, DK-5000 Odense C, Denmark. ${ }^{4}$ Department of Emergency Medicine and Department of Respiratory Medicine, Odense University Hospital, DK-5000 Odense C Denmark. ${ }^{5}$ Department of Emergency Medicine, Odense University Hospital, DK-5000 Odense C, Denmark. ${ }^{6}$ Hospital of South West Jutland, DK-6700 Esbjerg, Denmark.

Received: 2 May 2017 Accepted: 7 November 2017

Published online: 15 November 2017

\section{References}

1. Ranzani OT, Zampieri FG, Besen BA, Azevedo LC, Park M. One-year survival and resource use after critical illness: impact of organ failure and residual organ dysfunction in a cohort study in Brazil. Critical care (London, England) 2015;19:269.

2. Sakr Y, Lobo SM, Moreno RP, Gerlach H, Ranieri VM, Michalopoulos A, et al. Patterns and early evolution of organ failure in the intensive care unit and their relation to outcome. Critical care (London), Des Eng 2012;16(6):R222.

3. Lone NI, Walsh TS. Impact of intensive care unit organ failures on mortality during the five years after a critical illness. Am J Respir Crit Care Med. 2012;186(7):640-7.
4. $\quad$ Bingold TM, Lefering R, Zacharowski $K$, Meybohm $P$, Waydhas $C$, Rosenberger $P$, et al. Individual organ failure and concomitant risk of mortality differs according to the type of admission to ICU—a retrospective study of SOFA score of 23,795 patients. PLoS One. 2015;10(8):e0134329.

5. Churpek MM, Zadravecz FJ, Winslow C, Howell MD, Edelson DP. Incidence and prognostic value of the systemic inflammatory response syndrome and organ dysfunctions in ward patients. Am J Respir Crit Care Med. 2015;192(8):958-64

6. Benns M, Carr B, Kallan MJ, Sims CA. Benchmarking the incidence of organ failure after injury at trauma centers and nontrauma centers in the United States. The journal of trauma and acute care surgery. 2013;75(3):426-31.

7. Holler JG, Henriksen DP, Mikkelsen S, Rasmussen LM, Pedersen C, Lassen AT. Shock in the emergency department; a 12 year population based cohort study. Scandinavian journal of trauma, resuscitation and emergency medicine. 2016;24:87.

8. Angus DC, van der Poll T. Severe sepsis and septic shock. N Engl J Med. 2013;369(21):2063.

9. Jessen MK, Skibsted S, Shapiro NI. Number of organ dysfunctions predicts mortality in emergency department patients with suspected infection: a multicenter validation study. Eur J Emerg Med. 2015;24(3):176-82.

10. Shapiro N, Howell MD, Bates DW, Angus DC, Ngo L, Talmor D. The association of sepsis syndrome and organ dysfunction with mortality in emergency department patients with suspected infection. Ann Emerg Med. 2006:48(5):583-90. 90.e1

11. Rohde JM, Odden AJ, Bonham C, Kuhn L, Malani PN, Chen LM, et al. The epidemiology of acute organ system dysfunction from severe sepsis outside of the intensive care unit. J Hosp Med. 2013;8(5):243-7.

12. Chen YX, Li CS. Risk stratification and prognostic performance of the predisposition, infection, response, and organ dysfunction (PIRO) scoring system in septic patients in the emergency department: a cohort study. Critical care (London, Des Eng). 2014;18(2):R74.

13. Kakebeeke D, Vis A, de Deckere ER, Sandel MH, de Groot B. Lack of clinically evident signs of organ failure affects ED treatment of patients with severe sepsis. Int J Emerg Med. 2013;6(1):4

14. Guirgis FW, Brakenridge S, Sutchu S, Khadpe JD, Robinson T, Westenbarger $R$, et al. The long-term burden of severe sepsis and septic shock: sepsis recidivism and organ dysfunction. The journal of trauma and acute care surgery. 2016;81(3):525-32.

15. Moher D, Shamseer L, Clarke M, Ghersi D, Liberati A, Petticrew M, et al. Preferred reporting items for systematic review and meta-analysis protocols (PRISMA-P) 2015 statement. Systematic reviews. 2015:4:1.

16. Shamseer L, Moher D, Clarke M, Ghersi D, Liberati A, Petticrew M, et al. Preferred reporting items for systematic review and meta-analysis protocols (PRISMA-P) 2015: elaboration and explanation. BMJ (Clinical research ed). 2015;349:97647

17. Higgins JPT, Green, S. (editors). Cochrane handbook for systematic reviews of interventions version 5. 1. 0 [updated march 2011]. Available from http:// handbook-5-1.cochrane.org/: The Cochrane Collaboration; 2011.

18. Hayden JA, van der Windt DA, Cartwright JL, Cote P, Bombardier C. Assessing bias in studies of prognostic factors. Ann Intern Med. 2013;158(4):280-6

19. Wells GA, Shea B, O'Connell D, Peterson J, Welch V, Losos M, et al. The Newcastle-Ottawa scale (NOS) for assessing the quality of nonrandomised studies in meta-analyses. Available: Http://www.ohrica/programs/clinical_ epidemiology/oxfordasp. 2014.

20. Higgins JPT, Altman DG, Sterne JAC (editors). Chapter 8: assessing risk of bias in included studies. In: Higgins JPT, Green S (editors). Cochrane Handbook for Systematic Reviews of Interventions Version 5.1.0 (updated March 2011). The Cochrane Collaboration, 2011. Available from http://handbook-5-1.cochrane.org/.

21. Ryan R. Cochrane Consumers and Communication Review Group. Heterogeneity and subgroup analysis in Cochrane consumers and communication group reviews: planning the analysis at protocol stage. http://cccrg.cochrane.org. 2016.

22. Ryan R. Cochrane Consumers and Communication Review Group. Cochrane consumers and communication review group: data synthesis and analysis. http://ccrg.cochrane.org 2016.

23. Ryan R, Hill S. How to GRADE the quality of the evidence. Cochrane consumers and communication group, avaliable at http://cccrg.cochrane. org/author-resources. Version 3.0. 2016.

24. Guyatt G, Oxman AD, Akl EA, Kunz R, Vist G, Brozek J, et al. GRADE guidelines: 1. Introduction-GRADE evidence profiles and summary of findings tables. J Clin Epidemiol. 2011;64(4):383-94. 